
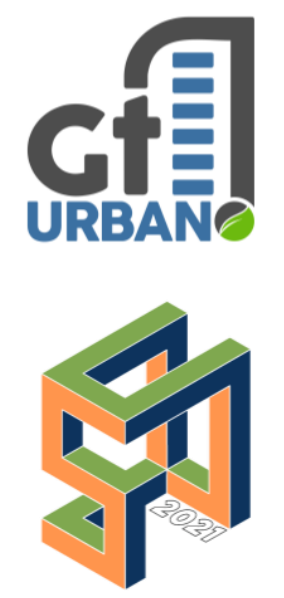

\section{SINGEURB}

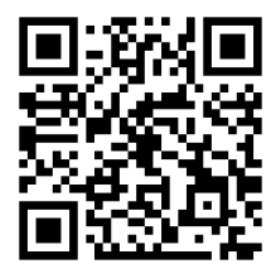

Como citar:

BALAGUER, Diogo Aoni; ALVES, Rosane Martins. Análise multicritério e uso de SIG para projetos com uso de energia solar fotovoltaica: um estudo bibliométrico. In: III SIMPÓSIO NACIONAL DE GESTÃO E

ENGENHARIA

URBANA:

SINGEURB, 2021,

Maceió. Anais...

Porto Alegre: ANTAC, 2021. p. 218225.

Disponível em: https://eventos.antac. org.br/index.php/sin geurb/issue/view/14

\title{
Análise multicritério e uso de SIG para projetos com uso de energia solar fotovoltaica: um estudo bibliométrico
} Multicriteria analysis and GIS use in solar photovoltaic energy projects: a bibliometric study

Diogo Aoni Balaguer, Universidade Federal do Rio de Janeiro, balaguer@poli.ufrj.br

Rosane Martins Alves, Universidade Federal do Rio de Janeiro, rosane.alves@poli.ufrj.br

\section{RESUMO}

A crescente preocupação com o meio ambiente torna a busca pela utilização de fontes renováveis de energia cada vez mais necessária, sendo fundamental a adoção de projetos mais sustentáveis e cada vez mais eficientes, principalmente no ambiente urbano. A utilização das diferentes ferramentas de análise multicritério, notadamente o método AHP (analytical hierarchy process), pode auxiliar na tomada de decisão em selecionar locais mais adequados para a implantação de painéis fotovoltaicos em projetos de geração de energia solar. De modo a traçar um panorama sobre as publicações científicas existentes na atualidade que abordem esta temática, este trabalho tem por objetivo a realização de um estudo bibliométrico para quantificar e identificar as tendências de pesquisa, desenvolvimento e evolução do tema. Foram selecionadas 72 publicações através de pesquisa na Base Scopus, sendo observado um crescimento no número de trabalhos publicados a partir de $2016 \mathrm{e}$ uma tendência de evolução da pesquisa e interesse no desenvolvimento de metodologias que combinem a utilização de ferramentas SIG (Sistema de Informações Geográficas) com a análise multicritério.

Palavras-chave: Análise Multicritério, Energia solar fotovoltaica, Estudo bibliométrico.

\begin{abstract}
The growing concern with the environment makes the search for the use of renewable energy sources more and more needed, making it essential to adopt more sustainable and efficient projects, especially in the urban environment. The use of different multicriteria analysis tools, notably the AHP (analytical hierarchy process) method, can help in decision making to select the most appropriate sites for the implementation of photovoltaic panels in solar energy generation projects. To provide an overview of current scientific publications that address this issue, this work aims to carry out a bibliometric study to quantify and identify trends in research, development, and the evolution of the subject. 72 publications were selected through research in the Scopus database, with an increase in the number of works published from 2016
\end{abstract}


onwards and a trend of research evolution and interest in the development of methodologies that combine the use of GIS tools (Geographical Information System) with the multicriteria analysis.

Keywords: Multicriteria analysis, Photovoltaic solar energy, Bibliometric study.

\section{INTRODUÇÃO}

Desde o acordo de Paris em 2015, o Brasil se comprometeu a adotar medidas para mitigar as mudanças climáticas, propondo uma meta de pelo menos $23 \%$ da matriz energética nacional advinda de fontes renováveis, exceto a hidroelétrica. A proximidade do Brasil com a linha do Equador permite uma distribuição uniforme de incidência solar e alta irradiação durante todo ano, tornando o país propício para a geração de energia fotovoltaica e com enorme potencial para o desenvolvimento desta tecnologia. A cada ano o preço da energia solar fotovoltaica diminui e a eficiência dos painéis é cada vez maior (MAGALHÃES et al., 2020).

A expansão da produção de energia através de tecnologias fotovoltaicas, segundo Thebault et al. (2020), vem aumentando bastante nos últimos anos. Em 2019, representava cerca de 2,6\% da produção de energia no mundo, devendo chegar a $25 \%$ da geração de eletricidade mundial por volta do ano de 2050, chegando a uma capacidade instalada de 8500 GW. Previsões apontam que $40 \%$ da capacidade de geração será proveniente de sistemas FV (fotovoltaicos) instalados em coberturas de edifícios, portanto, as cidades terão papel importante nesta transição de matrizes energéticas.

Magalhães et al. (2020) afirmam que, do ponto de vista ambiental, a escolha de energia solar fotovoltaica é justificada por não poluir o ar ou cursos hídricos e não emitir CO2. No entanto, os impactos associados com a produção dos painéis FV deve ser considerado. Outra vantagem da utilização da energia fotovoltaica é a área necessária para a geração $\left(\mathrm{m}^{2} / \mathrm{GWh}\right)$, sendo a menor dentre as fontes renováveis. A avaliação da adequabilidade dos locais de implantação do sistema FV deve considerar fatores ambientais, geomorfológicos, espaciais e climáticos.

Edificações aptas a receber instalações de painéis fotovoltaicos possuem características distintas, por este motivo, procedimentos para a classificação de acordo com a adequabilidade devem ser desenvolvidos. Vários fatores devem ser levados em consideração como a presença de superestruturas nos telhados, robustez estrutural, viabilidade econômica, patrimônios tombados e características estéticas das edificações (THEBAULT et al., 2020). Já para Al Garni e Awasthi (2018), os principais critérios para a classificação dos locais são: ambiental, espacial, climático, orográfico, social e riscos. Foram identificados 39 subcritérios, principalmente dentro do aspecto climático e espacial, e, o critério considerado o mais importante foi a quantidade de irradiação solar (AL GARNI; AWASTHI, 2018).

Apesar de focar principalmente nos aspectos econômicos, de viabilidade técnica e legais, critérios relacionados ao social e aos interesses da comunidade local devem ser considerados na análise. Para tal, Sward et al. (2021), propõem a inclusão de critérios socioeconômicos e demográficos aos métodos tradicionais de seleção de locais para a implantação de projetos em energia renovável, através de coleta de dados e pesquisas para o melhor entendimento das preferências de determinada comunidade.

De acordo com Al Garni e Awasthi (2018), a identificação de locais adequados para a instalação dos painéis FV é crucial para a avaliação da viabilidade do projeto, e a tecnologia SIG é uma ferramenta poderosa para 
consultar, analisar e editar dados, mapas e informações espaciais, auxiliando na escolha dos locais de implantação dos projetos e no planejamento energético. Nas últimas duas décadas, métodos MCDM (multicriteria decision-making) têm sido utilizados nas áreas de gerenciamento energético, avaliação de impactos ambientais, energias renováveis, sustentabilidade energética dentre outras (AL GARNI; AWASTHI, 2018). A combinação da tecnologia SIG e da análise multicritério tem ganhado relevância nos últimos anos. A maioria dos estudos nesta área utiliza a metodologia AHP para definir os pesos dos critérios (ZAMBRANOASANZA; QUIROS-TORTOS; FRANCO, 2021).

O desenvolvimento de modelos que integrem o SIG com a análise multicritério podem determinar localizações ideais para a captação de energia solar, aumentando a eficiência dos painéis FV, maximizando a geração de energia e minimizando os custos. Segundo Al Garni e Awasthi (2018), a seleção do local de implantação de um projeto FV é influenciada por diversos aspectos, portanto, a aplicação de métodos de análise multicritério pode auxiliar na tomada de decisão e em diversos projetos na área de planejamento energético.

Estudos que utilizam a metodologia da análise multicritério aliada a tecnologia SIG, segundo Sward et al. (2021), seguem um procedimento comum. Incialmente, são selecionadas as regiões, então é realizada uma revisão da literatura em conjunto com consultas a especialistas para determinar os critérios que serão adotados, se dividindo em critérios de exclusão e critérios de decisão. Muitos estudos também propõe uma análise de sensibilidade para verificar a solidez do método proposto (SWARD et al., 2021).

O AHP, de acordo com Al Garni e Awasthi (2018), é um dos métodos mais utilizados para classificar alternativas, combina critérios qualitativos e quantitativos, sendo reconhecido pela comunidade científica internacional como uma técnica flexível e robusta para facilitar a resolução de problemas complexos de tomada de decisão. Conforme Mokarram et al. (2020), a abordagem AHP é um método multicritério para a tomada de decisão que é capaz de desmembrar um problema complexo com diversos fatores em uma estrutura hierárquica, compondo níveis com seus respectivos atributos e pode também ser utilizado em conjunto com outros métodos. Tipicamente, os critérios de decisão dependem do objeto de estudo, acesso aos dados georreferenciados e literatura existente (AL GARNI; AWASTHI, 2018).

De modo a traçar um panorama sobre as publicações científicas existentes na atualidade que abordam a utilização das diferentes ferramentas de análise multicritério no auxílio da tomada de decisão na seleção de locais de implantação de painéis fotovoltaicos, este artigo tem por objetivo a realização de um estudo bibliométrico para quantificar e identificar as tendências de pesquisa, desenvolvimento e a evolução deste tema, devido à crescente relevância da utilização de energias renováveis no âmbito das áreas urbanas considerando o aumento da preocupação com o meio ambiente e a busca de projetos cada vez mais sustentáveis.

\section{METODOLOGIA}

Foi utilizada a bibliometria, técnica quantitativa e estatística que mede índices de produção e de disseminação do conhecimento, que permite acompanhar o desenvolvimento de diversas áreas científicas.

A base de dados selecionada para a pesquisa foi a Scopus devido à sua relevância em publicações relacionadas à temática pesquisada. Na seleção dos artigos foram utilizados termos e operadores booleanos conforme a sintaxe: "mcdm OR multicriteria OR multiple-criteria OR multi-criteria AND photovoltaic OR 
pv AND site AND NOT water AND NOT wind", buscando em títulos dos artigos, resumos e palavraschave.

De modo a limitar a pesquisa à área de energia solar fotovoltaica, foram excluídos estudos que tratassem de outras fontes de energia renovável como a eólica e projetos de abastecimento de água.

Optou-se pela não limitação do período da pesquisa, por ser um assunto relativamente novo e de modo a estabelecer uma evolução dos trabalhos publicados, fornecendo dados de artigos a partir do ano de 2008, ano das primeiras publicações relevantes no tema, até 2021.

As publicações selecionadas foram analisadas quanto à quantidade de trabalhos publicados por ano, tipos de documentos, autores mais proeminentes na área de estudo, locais onde foram feitas as publicações, periódicos mais relevantes no tocante ao número de publicações, artigos mais relevantes considerando o número de citações e os focos principais das pesquisas com base nas palavras-chave mais utilizadas através da inserção dos dados dos artigos no software VOSviewer.

\section{RESULTADOS E DISCUSSÕES}

De acordo com a metodologia proposta, foram selecionados 72 trabalhos publicados na Base Scopus, dentre eles, 47 artigos, 16 trabalhos publicados em conferências, 6 revisões bibliográficas, além de 3 capítulos de livros.

A Figura 1 apresenta a quantidade de documentos publicados por ano, sendo possível observar uma tendência de incremento no número de publicações a partir do ano de 2016, desde os primeiros trabalhos publicados em 2008. Apesar da queda nas publicações observada no ano de 2018, os últimos anos apontam um interesse crescente em pesquisa sobre o tema.

Figura 1 - Publicações por ano

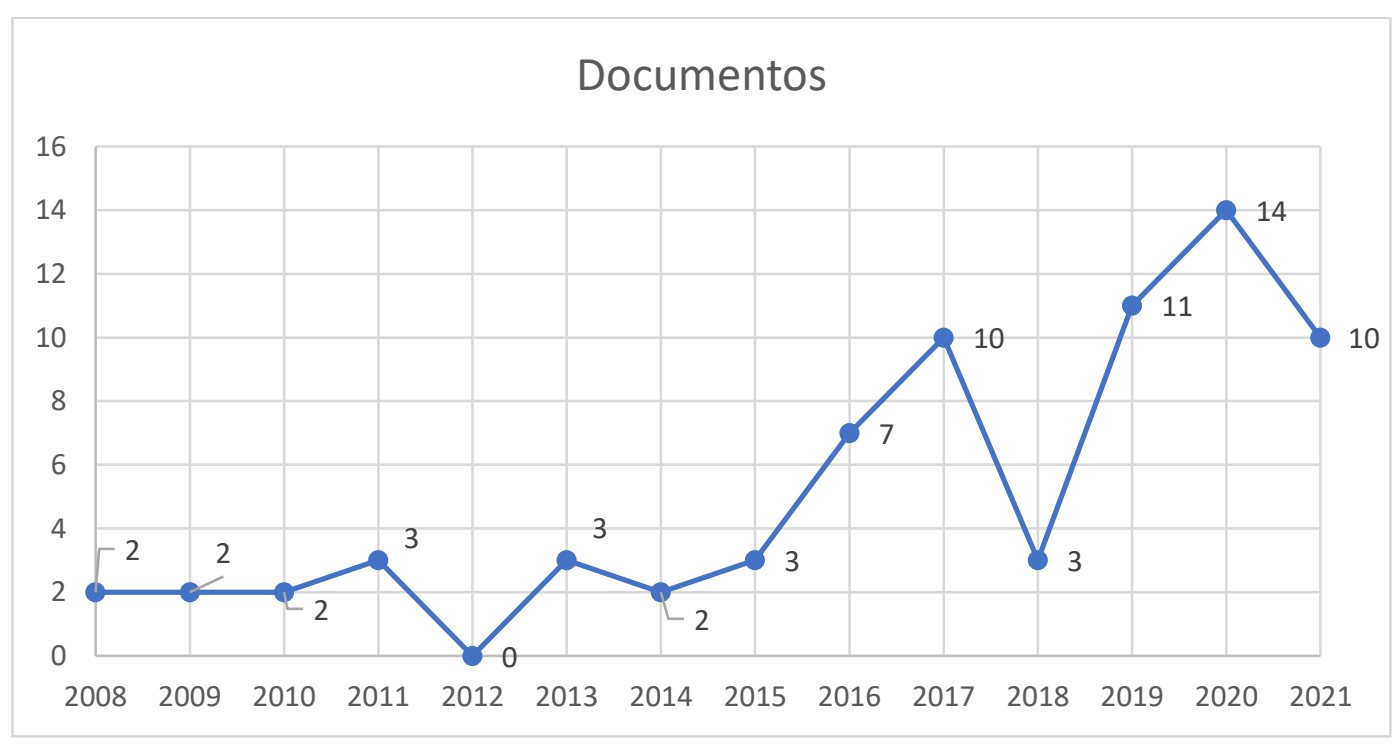

Fonte: Os autores 
Dentre os autores, destacam-se Al Garni, e Awasthi, com 4 trabalhos cada, bem como Sánchez-Lozano, com 3 publicações. Além destes, diversos outros autores aparecem com 2 publicações, conforme Figura 2. Dos periódicos que publicaram artigos, cerca de $40 \%$ dos trabalhos selecionados, são relacionados à área de eficiência energética e sustentabilidade, dentre eles, Renewable Energy, Renewable And Sustainable Energy Reviews, Journal Of Cleaner Production, Energies, Energy, Applied Energy e Energy Conversion And Management.

Figura 2 - Publicações por autor

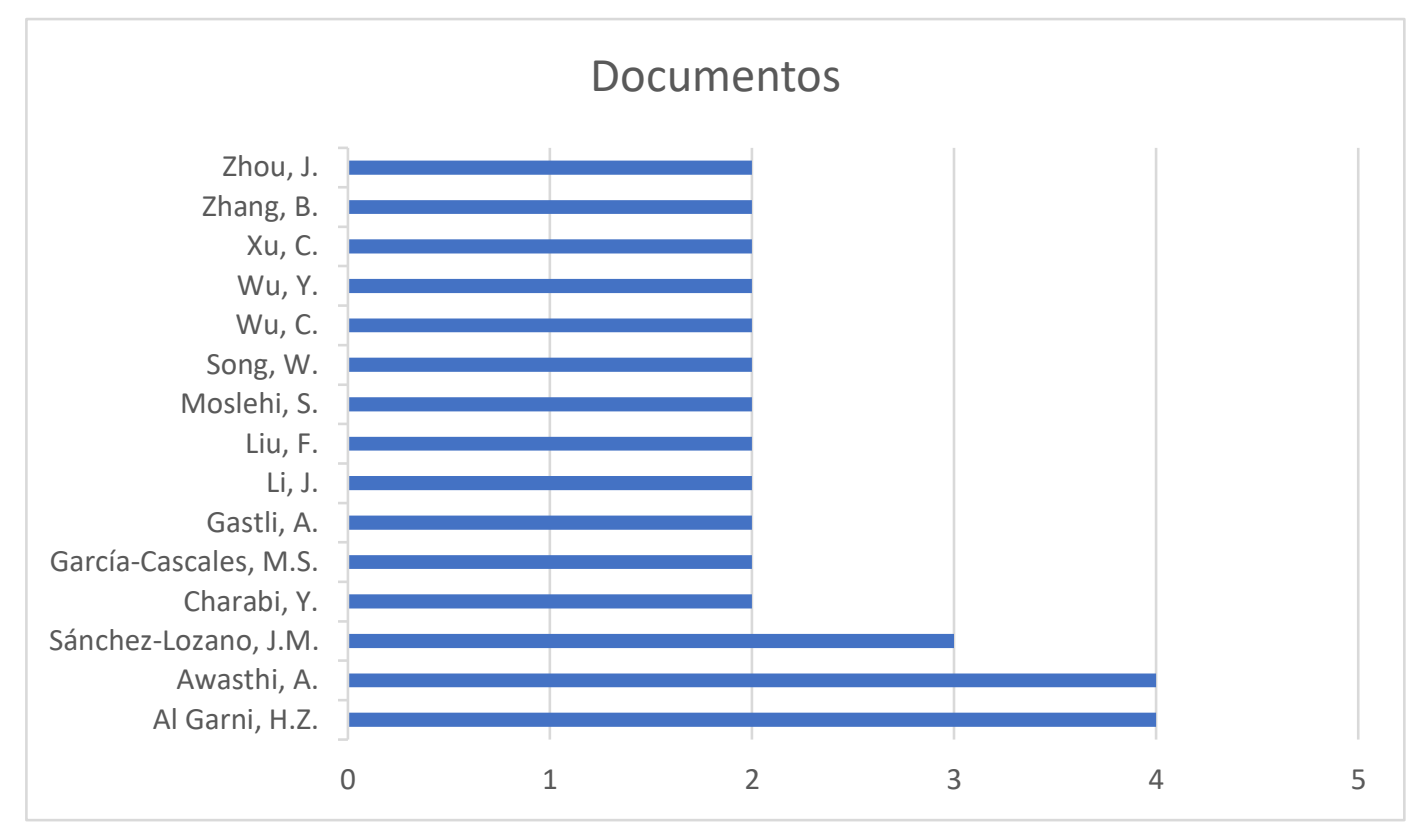

Fonte: Os autores

Já no aspecto da distribuição geográfica dos trabalhos publicados, as regiões da América do Norte, Europa, sudeste da Ásia e norte da África são importantes polos de desenvolvimento de pesquisa na área de análise multicritério aplicada à seleção de locais para a implantação de projetos em energia solar fotovoltaica, como pode ser observado na Figura 3. 
Figura 3 - Publicações por país

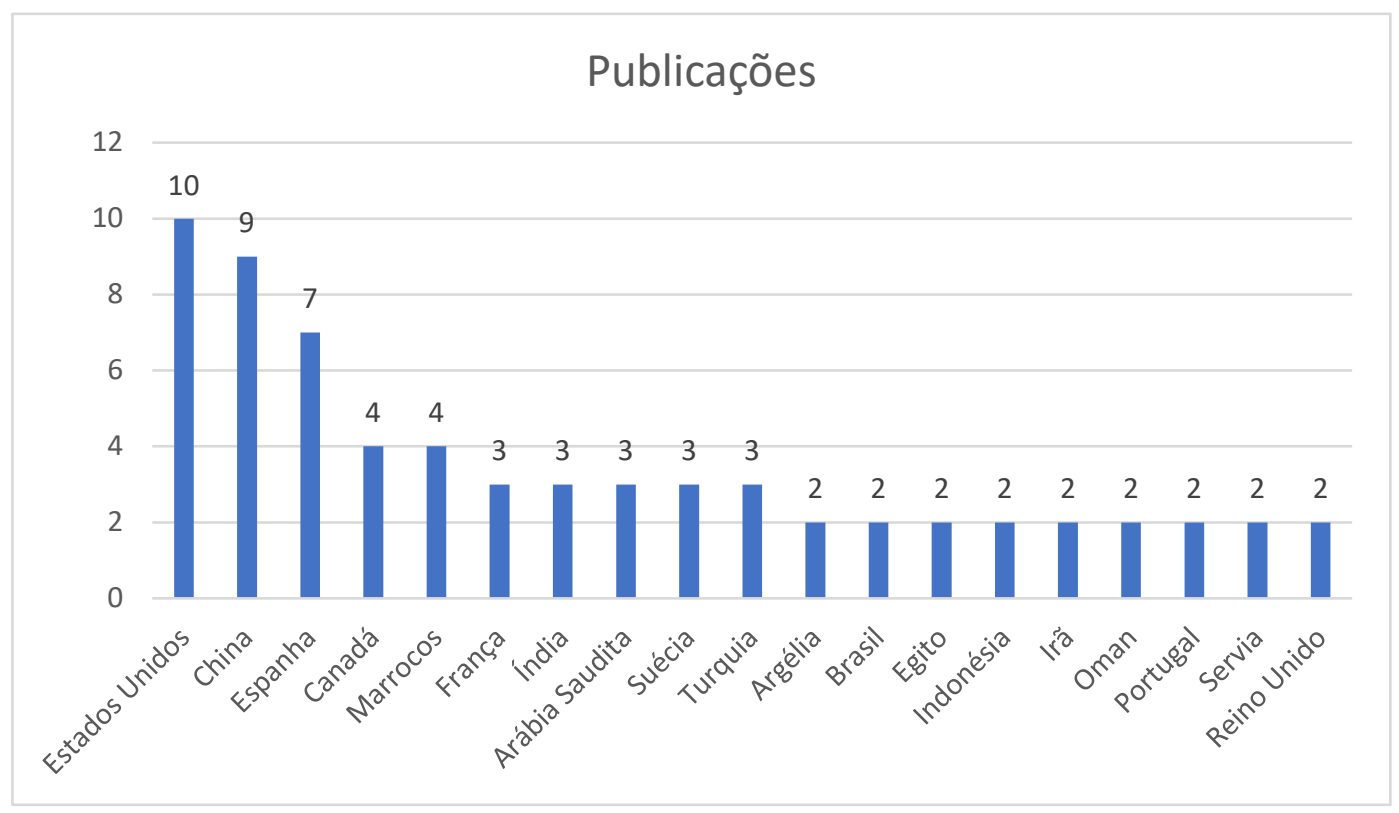

Fonte: Os autores

O Brasil atualmente aparece com 2 publicações (UTFPR e UNESP), o que representa uma grande oportunidade para crescimento e necessidade do desenvolvimento de pesquisas nesta área de estudo, em virtude do seu alto potencial energético. A Figura 4 apresenta a distribuição geográfica das publicações.

Figura 4 - Distribuição Geográfica das publicações
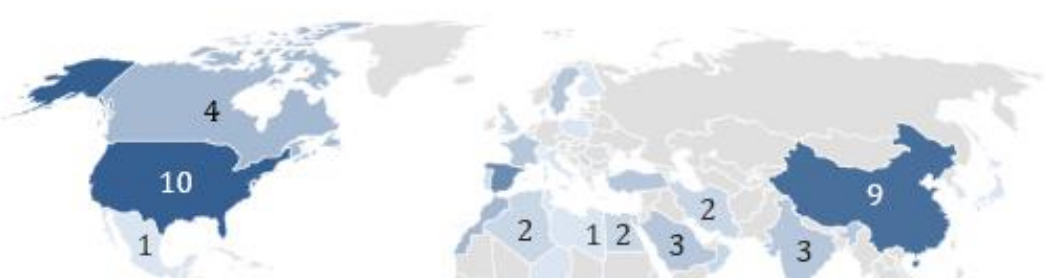

Publicações

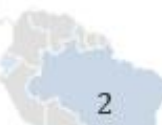

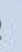

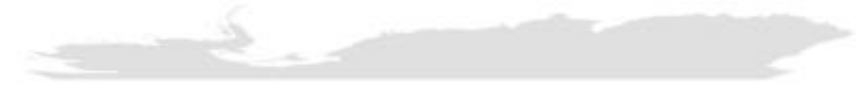


A utilização do SIG aliada às ferramentas de análise multicritério foi abordada em 55 dos trabalhos selecionados para este estudo, ou seja, $76 \%$ das publicações, principalmente fornecendo subsídio para o estabelecimento de critérios ambientais, climáticos, orográficos e de localização (ELKADEEM et al., 2021). Além da utilização conjunta entre SIG e os métodos de análise multicritério, os artigos analisados tiveram como foco de pesquisa, considerando a incidência dos termos nas diversas publicações, a seleção, energia, tecnologia e custo, de acordo com o mapa de calor de palavras-chave elaborado com o auxilio do software VOSviewer.

Figura 5 - Termos mais utilizados

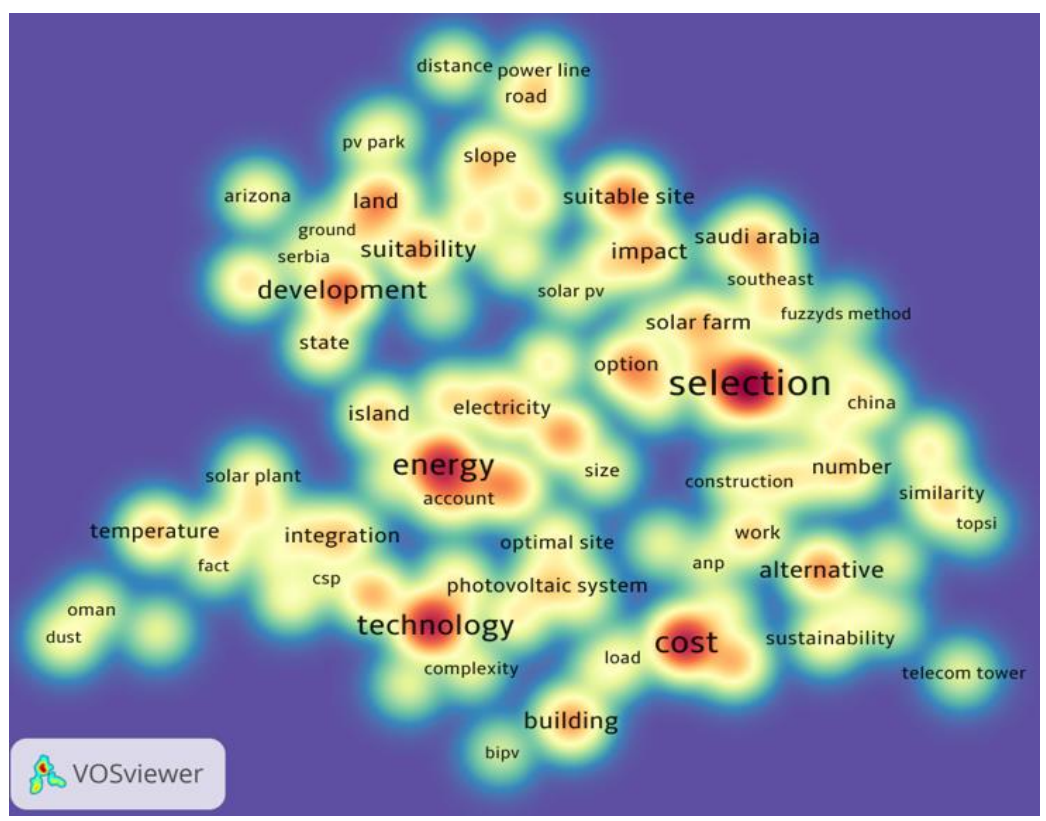

Fonte: Os autores

\section{CONSIDERAÇÕES FINAIS}

A partir dos 72 artigos selecionados, no período entre os anos de 2008 e 2021, foi observado um aumento na quantidade de publicações a partir de 2016, bem como uma tendência de incremento no interesse e no desenvolvimento de pesquisas relacionadas ao tema nos anos seguintes. No quantitativo de publicações destacam-se: América do Norte, Europa, sudeste da Ásia e norte da África. O Brasil aparece com 2 publicações, apresentando alto potencial para o desenvolvimento de pesquisas, podendo aumentar este número e futuramente figurar entre países de maior evidência.

As publicações tiveram como foco principal a seleção de locais para a implantação de painéis FV, desenvolvimento de tecnologias, busca de eficiência energética e redução de custos, conforme maior recorrência dos termos nas publicações. A utilização em conjunto do SIG com metodologias de análise multicritério, tratada em $76 \%$ dos trabalhos, monstra sua importância no estabelecimento de critérios para a seleção de locais mais adequados para implantação de projetos com uso de energia solar fotovoltaica. 
É evidente a escassez de estudos voltados para a produção científica brasileira na área pesquisada. A intensificação de estudos e publicações irão contribuir com a elaboração de políticas e estratégias para superar carências e limitações, buscando maior desenvolvimento científico, tecnológico e econômico.

Considerando o aumento da preocupação com o meio ambiente e a necessidade de se fomentar a utilização de energias renováveis além da concepção de projetos cada vez mais eficientes do ponto de vista energético, principalmente no ambiente urbano, pode-se vislumbrar uma tendência geral no aumento do interesse e no desenvolvimento de pesquisas no tema em questão nos próximos anos.

\section{REFERÊNCIAS}

AL GARNI, H. Z.; AWASTHI, A. Solar PV Power Plants Site Selection: A Review. In: ADVANCES in Renewable Energies and Power Technologies Solar PV Power Plants Site Selection: A Review. Montreal, QC, Canada: Elsevier, 2018. v. 1: Solar and Wind Energies, cap. 2, p. 57-75.

ELKADEEM, M. R.; YOUNES, A.; SHARSHIR, S. W.; CAMPANA, P. E.; WANG, S. Sustainable siting and design optimization of hybrid renewable energy system: A geospatial multi-criteria analysis. Applied Energy, n. 295, p. 1-35, 2021.

MAGALHÃES, I. B.; NOGUEIRA, G. C.; ALVES, I. S.; CALIJURI, M. L.; LORENTZ, J. F.; ALVES, S. C. Site suitability for photovoltaic energy expansion: A Brazilian's high demand states study case. Remote Sensing Applications: Society and Environment, 2020.

MOKARRAM, M.; MOKARRAM, M. J.; GITIZADEH, M.; NIKNAM, T.; AGHAEI, J. A novel optimal placing of solar farms utilizing multi-criteria decision making (MCDA) and feature selection. Journal of Cleaner Production, 2020.

THEBAULT, M.; CLIVILLÉ, V.; BERRAH, L.; DESTHIEUX, G. Multicriteria roof sorting for the integration of photovoltaic systems in urban environments. Sustainable Cities and Society, v. 60, 2020.

SWARD, J. A.; NILSON, R. S.; KATKAR, V. V.; STEDMAN, R. C.; KAY, D. L.; IFFT, J. E.; ZHANG, K. M. Integrating social considerations in multicriteria decision analysis for utility-scale solar photovoltaic siting. Applied Energy, 2021.

ZAMBRANO-ASANZA, S.; QUIROS-TORTOS, J.; FRANCO, J. F. Optimal site selection for photovoltaic power plants using a GIS-based multi-criteria decision making and spatial overlay with electric load. Renewable and Sustainable Energy Reviews, v. 143, 2021. 